\title{
CIF: FERRAMENTA DE AVALIAÇÃO E REGULAÇÃO CLÍNICO-ASSISTENCIAL NO NASF-AB
}

ICF: Clinical assistance assessment and regulation tool at NASF-AB

Luana Padilha da Rocha, luanapadilha1895@gmail.com, http://lattes.cnpq.br/4844900203755742, Fisioterapeuta do Nasf-AB da Prefeitura da Cidade do Recife, Mestre em Enfermagem e Educação em Saúde, ORCID: 0000-0002-7879-8708;

Maria Soraida Silva Cruz, soraida.to@gmail.com, http://lattes.cnpq.br/1370862699105564, terapeuta Ocupacional do Nasf-AB da prefeitura da cidade do Recife, Mestre em saúde da criança e do adolescente, ORCID:0000-0001-6894-9536;

Janaína Gabriela Coêlho de Araújo, janagca_nutri@yahoo.com.br, http://lattes.cnpq.br/2787660421907980, nutricionista do Nasf-AB da Prefeitura da Cidade do Recife, Mestre em Gerontologia, ORCID:0000-0003-3505-3797;

Bruna Leitão Miranda, bruna_leitao@yahoo.com.br, http://lattes.cnpq.br/5482225436528972, Psicóloga do Nasf-AB da Prefeitura da Cidade do Recife, Especialista em Psicologia, ORCID: 00000002-5588-0362;

Robélia do Nascimento Lopes, robelia.lopes@ufpe.br, http://lattes.cnpq.br/4776411589540046, Assistente Social do Nasf-AB da Prefeitura da Cidade do Recife, Especialista em Políticas de Proteção Social, ORCID: 0000-0002-0289-9713;

Suênia Xavier Gonçalves, sueniaxavier83@gmail.com, http://lattes.cnpq.br/4645757816772564, Fisioterapeuta do Nasf-AB da Prefeitura da Cidade do Recife, Mestre em Saúde Pública, ORCID:0000-0001-5846-6338;

Aline Fernanda de Andrade, fga.alineandrade@gmail.com, http://lattes.cnpq.br/8223730598536647, Fonoaudióloga do Nasf-AB da Prefeitura da Cidade do Recife, Especialista em Saúde da Família, ORCID: 0000-0002-3767-908;

José Candido de Araújo Filho, josecandidofilho@gmail.com, http://lattes.cnpq.br/7063014238461302, docente da Faculdade de Comunicação e Turismo de Olinda, do Centro Universitário Facol e do Centro Universitário Estácio do Recife, Mestre em Fisioterapia, ORCID: 0000-0002-3941-3043.

Autora correspondente: Luana Padilha da Rocha R. Prof. Fernando Mota, 217 - Ibura de baixo, Recife - PE, 51230-250 8198874-2907 | luanapadilha1895@gmail.com

\section{RESUMO}

Este artigo objetiva descrever a experiência de um Nasf-AB na construção de um instrumento de avaliação e regulação clínico-assistencial, tendo a CIF como alicerce. O estudo consiste num relato de experiência descritivo e retrospectivo de uma equipe Nasf-AB do Recife. $O$ instrumento produzido foi estruturado em duas partes: a primeira sintetiza informações da anamnese e a segunda é um checklist com 27 códigos elencados da CIF, dos quais cinco foram destacados numa subescala que analisa mobilidade e autocuidado. $\mathrm{O}$ instrumento baseado na CIF favoreceu uma linguagem comum entre profissionais da equipe, podendo ser aplicável às demandas assistenciais acompanhadas, permitiu aprimorar o acompanhamento interdisciplinar, na perspectiva da integralidade e organização do suporte clínico-assistencial, obedecendo aos pressupostos da coordenação e gestão compartilhada do cuidado, traçando tipos de acompanhamento, critérios de encaminhamento e alta. Sugere-se que o instrumento possa ser objeto de validação futura entre especialistas, tornando-se alternativa para regulação assistencial do Nasf-AB.

Descritores: Atenção Primária à Saúde. Saúde da Família. Classificação Internacional de Funcionalidade, Incapacidade e Saúde. 


\section{ABSTRACT}

This article aims to describe the experience of a Nasf-AB in the construction of a clinical care assessment and regulation instrument, having the ICF as its foundation. The study consists of a descriptive and retrospective experience report of a Nasf-AB team in Recife. The instrument produced was structured in two parts: the first synthesizes information from the anamnesis and the second is a checklist with 27 codes listed from the ICF, five of which were highlighted in a subscale that analyzes mobility and self-care. The instrument based on the ICF favored a common language among the team's professionals, which could be applicable to the followed care demands, allowed the improvement of the interdisciplinary monitoring, from the perspective of comprehensiveness and organization of clinical and care support, obeying the assumptions of coordination and shared management of care, outlining types of follow-up, referral and discharge criteria. It is suggested that the instrument may be subject to future validation among specialists, becoming an alternative for healthcare regulation by the Nasf-AB.

Descriptors: Primary Health Care. Family Health. International Classification of Functioning, Disability and Health.

\section{INTRODUÇÃO}

O Núcleo de Apoio à Saúde da Família foi criado em 2008 com o intuito de aumentar a resolutividade e o escopo das ações da Atenção Primária à Saúde (APS) no Brasil. Hoje nomeado de Núcleo Ampliado de Saúde da Família e Atenção Básica (Nasf-AB), caracteriza-se por ser uma equipe multiprofissional que desenvolve atividades conjuntas com as equipes de Saúde da Família (eSF) de forma integrada e colaborativa. A fim de potencializar a cooperação interdisciplinar e aumentar a capacidade resolutiva da APS, tem como um de seus pressupostos de trabalho a lógica do Apoio Matricial (AM), que envolve a atuação conjunta das duas equipes, em atividades de natureza técnico-pedagógica e clínicoassistencial, visando a integralidade do cuidado ${ }^{1}$.

Considerando que o fazer do Nasf-AB é integrado com as eSF, com o foco prioritário nas ações de promoção e prevenção da saúde, sua finalidade é ampliar o leque de ofertas e incrementar o arsenal tecnológico do primeiro nível de contato da Rede de Atenção à Saúde (RAS), sem configurar serviço de acesso direto, porém contribuindo para melhoria da saúde e do bem-estar².

$O$ Nasf-AB tem um arranjo organizacional e assistencial inovador, cuja complexidade de implementação impõe importantes especificidades entre os modos de operar no país e tem suscitado discussões sobre suas finalidades e seu lugar na RAS. Soma-se a isso o fato de as equipes não terem seus processos de trabalho plenamente sistematizados, o que torna escassos os estudos que versam sobre a análise das características desses processos ${ }^{2-4}$.

Mesmo sendo um dispositivo que contribui para ampliação do acesso na APS, com potencial para influenciar o modo como as equipes produzem cuidado e se relacionam com outros serviços, o Nasf-AB enfrenta desafios que comprometem a integralidade: carência de serviços especializados e dificuldade quanto a sua disponibilidade e modo de funcionamento; escassez de definições teóricas orientadoras da sua atuação; pouca clareza sobre suas atribuições e forma de inserção na APS nos documentos norteadores; fragilidades na organização do trabalho entre as equipes; e inexistência de critérios de encaminhamentos e informalidade dos fluxos $2,3,5$.

No âmbito clínico-assistencial, observa-se um distanciamento no desenvolvimento do cuidado longitudinal e situações de descontinuidade terapêutica, inclusive no que diz respeito à articulação com outros níveis de atenção da RAS, além de uma pouca contribuição na amplitude e resolutividade dos atendimentos clínicos individuais e na qualificação dos encaminhamentos para outros serviços, demonstrando uma fragilidade do Nasf-AB² . 
O Nasf-AB tem como propósito atuar de forma compartilhada junto às eSF para superar a fragmentação, objetivando a construção de redes de atenção e cuidado, colaborando com a observância do princípio da integralidade ${ }^{6}$. A metodologia do AM, enquanto um modo de qualificar o cuidado, pode reduzir a quantidade de encaminhamentos desnecessários da APS e facilitar o compartilhamento do cuidado ${ }^{3}$.

O funcionamento do AM e da equipe de referência depende de arranjos organizacionais e formas de gestão do trabalho que ampliem as possibilidades de diálogo entre diferentes especialidades e a prática da Clínica Ampliada (CA), sendo essa uma ferramenta que permite a compreensão de outros aspectos do sujeito pelos profissionais de saúde, apontando para uma transformação da atenção individual e coletiva 7. Nesse sentido, o fazer do Nasf-AB vinculase ao conceito da Classificação Internacional da Funcionalidade, Incapacidade e Saúde (CIF).

A CIF é um modelo biopsicossocial de funcionalidade com informação em saúde, configurando uma ferramenta epidemiológica de classificação da saúde a nível individual e populacional. Baseia-se em evidências e desenvolvimento de programas que avaliem a eficiência, eficácia e efetividade das ações no âmbito da saúde coletiva ${ }^{8}$. Associada ao AM e a CA, apresenta-se como instrumento potencial para pesquisa, clínica, planejamento e gestão em saúde, sendo importante na identificação do estado de funcionalidade da população ${ }^{9}$.

Pensar no fazer do Nasf-AB remete à compreensão do seu objeto de atuação, dos modos de organização da produção de seus serviços e de seus instrumentos de trabalho ${ }^{10}$. A inexistência de diretrizes municipais para construção de um processo de trabalho peculiar nos locais onde as equipes foram inseridas dá às equipes Nasf-AB uma maior autonomia na construção de ações e possibilidades de acordo com as necessidades e potencialidades de cada território, trazendo singularidade e inovação para esse processo de trabalho ${ }^{11}$.

Diante do exposto, considerando o papel do Nasf-AB na retaguarda assistencial às eSF, a carência de instrumentos, normativas e diretrizes que orientem seu processo de trabalho com vistas a gerir e coordenar o cuidado dos usuários, acionar a RAS de modo racional e oportuno, e tendo a CIF como alicerce para o cuidado integral em saúde, este artigo tem como objetivo descrever a experiência de uma equipe Nasf$A B$ (eNasf) na construção de um instrumento de avaliação e regulação clínico-assistencial no âmbito da APS.

\section{METODOLOGIA}

O presente estudo consiste num relato de experiência descritivo e retrospectivo de uma eNasf da Cidade do Recife, composta por 7 profissionais das seguintes categorias: Fisioterapia, Fonoaudiologia, Nutrição, Psicologia, Serviço Social e Terapia Ocupacional. Esta eNasf encontra-se no mesmo território desde fevereiro de 2014 e apoia, atualmente 9 eSF, distribuídas em três Unidades Básicas de Saúde. Os dados que embasaram o presente relato foram produzidos entre dezembro de 2018 e fevereiro de 2019 e a sistematização do processo vivido ocorreu em quatro tempos, tomando como base o papel de construção do conhecimento e do aprimoramento das práticas individuais e coletivas que a sistematização das experiências exerce, ${ }^{12}$ conforme ilustrado na Figura 01.

\section{FIGURA 1. PROCESSO DE REALIZAÇÃO E SISTEMATIZAÇÃO DA EXPERIÊNCIA [Anexo]}

Fonte: adaptado de Holliday, $2019^{13}$ 


\section{RELATO DA EXPERIÊNCIA}

Ponto de partida: analisando os problemas

Desde o início de sua atuação, a demanda pelo suporte clínico-assistencial foi algo cotidiano na rotina da eNasf deste estudo, principalmente pela dificuldade de acesso dos usuários aos serviços de atenção especializada.

Apesar de ter um fluxo estabelecido de mapeamento das demandas por atendimentos, através do qual os casos que não configurassem urgência eram discutidos mensalmente nos espaços de reunião ordinária junto às eSF, havia uma lacuna no que diz respeito a uma diretriz para a definição do modo de acompanhamento do usuário no território, incluindo periodicidade de atendimentos por consultas nas unidades de saúde ou por visitas domiciliares, critérios de acompanhamento e alta, além da falta de direcionamentos que pudessem indicar a necessidade de encaminhamento para outros serviços da rede de saúde.

O resultado disso era uma grande variedade na forma de condução dos casos entre os profissionais da eNasf e até de um mesmo profissional, o que gerava discrepância na assistência prestada. Além disso, existiam muitos usuários em acompanhamento e que extrapolava a capacidade instalada da equipe 0 que resultava em uma descontinuidade no cuidado. Dessa forma, a eNasf precisou realizar uma análise sistêmica das suas ações assistenciais, repensando esse fazer e buscando propor um instrumento que pudesse auxiliar na definição de um modelo assistencial que possibilitasse uma linguagem única entre os profissionais do Nasf-AB. Foi a partir daí que, no final de 2018, a eNasf, durante suas oficinas de planejamento, iniciou um estudo direcionado para essa necessidade, buscando referências, instrumentos e normativas que pudessem embasar a elaboração de tal instrumento.
Elaboração do instrumento: proposição de uma ferramenta interprofissional

A análise dos casos acompanhados já evidenciava o grande leque de demandas que se apresentavam à eNasf, ressaltando que uma mesma situação precisava dos olhares das diferentes categorias profissionais que compunham a equipe. A multidimensionalidade envolvida nessas questões exigia que o instrumento a ser elaborado considerasse as prerrogativas da clínica ampliada. O objetivo desse momento era pensar num instrumento de fácil aplicação por qualquer profissional da eNasf, que contemplasse as diferentes especialidades dessa equipe, a fim criar critérios de acompanhamento, que norteassem a coordenação do cuidado e a continuidade da assistência ao longo do tempo, e de alta dos casos, devido a enorme demanda existente das eSF assistidas. A CIF foi então eleita como base para tal elaboração, visto que ela tem um caráter multidimensional e possibilita uma abordagem a partir das diversas condições de saúde que envolvem um indivíduo.

Nessa fase de construção do instrumento, buscou-se, inicialmente, dividi-lo em duas partes: a primeira composta por dados pessoais, de cobertura pela eSF, antecedentes pessoais/familiares, condições socioeconômicas, rede de apoio e demais informações colhidas durante uma anamnese; e a segunda parte, composta por um checklist de avaliação, contendo códigos da CIF com seus qualificadores.

Por não ser um instrumento conhecido por todas as profissionais da eNasf, foi preciso inicialmente fazer um matriciamento sobre a CIF, a fim de nivelar o conhecimento. Posterior a esse momento e no intuito de elencar os códigos da CIF a serem utilizados no instrumento, as categorias profissionais da eNasf fizeram um trabalho de mapeamento de códigos que pudessem refletir as demandas de núcleo, porém que permitissem sua compreensão e análise por 
outros profissionais. Isso era essencial para que o instrumento tivesse como característica a possibilidade de aplicação por qualquer trabalhador da eNasf. O resultado desse mapeamento foi levado para discussão em reuniões da eNasf para validação entre seus membros e compilação dos códigos num único instrumento.

Definindo tipos de acompanhamento $e$ critérios de encaminhamento, seguimento e alta

Findada a etapa de elaboração do instrumento, a eNasf começou a elaborar possíveis tipos de acompanhamentos dos casos no território, critérios para encaminhamento para outros serviços da rede de atenção à saúde, além de delinear critérios para alta da retaguarda do Nasf-AB.

Uma vez definidos os códigos da CIF para compor o instrumento, os tipos de acompanhamento e os critérios de encaminhamento e alta, a equipe precisou estabelecer uma forma de, a partir do preenchimento do checklist de avaliação, definir pontuações e fluxos que orientassem o seguimento dos casos pela eNasf. Foram aplicados alguns estudos pilotos e, após 4 versões do fluxograma de decisão, chegou-se ao modelo final de cálculo. A partir daí, definiuse a versão final do instrumento intitulado "Protocolo de elegibilidade para avaliação e monitoramento de casos".

O instrumento foi levado para discussão nas reuniões com as eSF apoiadas, com o intuito de apresentá-lo e ouvir as opiniões das equipes de referência, considerando que o trabalho na APS prima pela lógica interprofissional e o Nasf-AB tem como pressuposto gerir os casos acompanhados de modo compartilhado com essas equipes.

\section{Ponto de chegada}

A primeira versão do instrumento foi estruturada em 2 partes, conforme ilustrado na Figura 2. A primeira parte, de acordo com o descrito anteriormente, sintetiza informações colhidas durante a anamnese do usuário. Já a segunda parte do instrumento, organizada em formato de checklist, é composta por 27 códigos elencados da CIF, dos quais 5 foram destacados e representam uma subescala que analisa a mobilidade e o autocuidado do usuário.

\section{FIGURA 2. PROTOCOLO DE ELEGIBILIDADE PARA AVALIAÇÃO E MONITORAMENTO DE CASOS [Anexo]}

Fonte: elaborado pelas autoras, 2019

Cada categoria do checklist possui 7 alternativas de preenchimento que correspondem aos qualificadores utilizados na CIF: 0 - nenhuma deficiência/dificuldade; 1 - deficiência/dificuldade leve; 2 - deficiência/dificuldade moderada; 3 - deficiência/dificuldade grave; 4 deficiência/dificuldade completa; 8 - não especificada; 9 - não aplicável.

Cada qualificador tem um peso específico e norteia o cálculo para definir o perfil de acompanhamento, conforme ilustrado na figura 3 .

\section{FIGURA 3. FLUXOGRAMA DE DECISÃO PARA ELEIÇÃO DO TIPO DE ACOMPANHAMENTO DO USUÁRIO [Anexo]}

Fonte: elaborado pelas autoras, 2019 
O cálculo é baseado na proporção total de qualificadores selecionados entre 0 e 4 no checklist. Esse total entre 0 e 4 representará - escore geral da avaliação, totalizando $100 \%$. Quando $75 \%$ ou mais desse escore geral é de qualificador 0 , a forma de acompanhamento estabelecido será do tipo I, que se caracteriza por orientações à eSF (matriciamento) para manejo desses casos, bem como orientações para o usuário, família e/ou cuidadores, uma vez que o paciente avaliado apresenta uma boa condição funcional.

Caso o total de qualificadores 0 seja menor que $75 \%$, deve-se diminuir do escore geral a quantidade de qualificadores 0 e 0 restante (soma dos qualificadores de 1 a 4) gera um segundo score que representará 0 novo escore geral (100\%). Se desse novo escore, houver $75 \%$ ou mais de qualificadores 1, o acompanhamento tipo 2 será instituído. Este acompanhamento é representado por um seguimento mais sistemático da eNasf, com realização de retornos de atendimento mensais. Fazem parte desse grupo: usuários com limitação funcional leve que não interfira significativamente nas Atividades de Vida Diária (AVD), Atividades Instrumentais de Vida Diária (AIVD) e atividades laborais e em situação individual/ambiental que não impeça seguimento das orientações no domicílio.

Por outro lado, se do segundo escore geral houver preenchimento de menos de $75 \%$ de qualificadores 1 , subtrai-se daquele a quantidade destes qualificadores e o restante (soma dos qualificadores de 2 a 4) gera um terceiro escore que será o novo escore geral $(100 \%)$. Se desse novo escore, houver $75 \%$ ou mais de qualificadores 2, o acompanhamento do usuário também será tipo 2 (seguimento mais sistemático da eNasf), contudo, diferenciando do anterior, a realização dos retornos de atendimento será quinzenal. Fazem parte desse grupo usuários com limitação funcional moderada que interfere nas atividades cotidianas (semidependência para AVD, AIVD e atividades laborais) e em situação individual/ambiental que não impeça seguimento das orientações no domicílio.

Seguindo o fluxograma de decisão, se do terceiro escore geral, menos de $75 \%$ do preenchimento seja de qualificadores 2 , partese para a subescala do instrumento. Se dos 5 códigos, $25 \%$ ou menos dos qualificadores forem 3, o acompanhamento será do tipo 3, que se configura como orientação aos cuidadores e referência para serviço especializado. Fazem parte dessa categoria os usuários com graves limitações funcionais e/ou dependência total nas AVD, que apresentem lesões complexas, tratadas cirurgicamente ou não, com comorbidades associadas, situação socioeconômica e/ou ambiental que não impeçam seu deslocamento ao serviço de reabilitação e com indicação de iniciar o tratamento o mais rápido possível para evitar complicações, sequelas ou incapacidades.

Já se dos 5 códigos da subescala, mais de $25 \%$ forem de qualificadores 4 , o acompanhamento será do tipo 4 , definido como orientação aos cuidadores e/ou referência para o serviço de atendimento domiciliar (SAD). Encaixam-se nesse grupo usuários com graves limitações funcionais que apresentem lesões complexas, tratadas cirurgicamente ou não, com comorbidades associadas. $O$ que diferencia do tipo 3 é a situação socioeconômica e/ou ambiental que impeça os usuários no seu deslocamento ao serviço de especializado.

Se na subescala não houver preenchimento de códigos 3 ou 4, o usuário ficará no acompanhamento tipo 2, com seguimento quinzenal ou mensal, a depender da avaliação compartilhada entre eNasf e eSF, durante a discussão do caso/situação nos espaços de reunião ordinária entre as equipes.

Para definição de alta do acompanhamento do Nasf-AB foram estipulados 4 parâmetros: 
- Por objetivos atingidos: usuários que evoluíram positivamente, de acordo com o prognóstico funcional e com a avaliação e objetivos traçados pela eNasf;

- Por objetivos não atingidos: usuários que não evoluíram positivamente de acordo com o prognóstico funcional e com a avaliação e objetivos traçados pela eNasf por falta de adesão e dificuldade de seguimento das orientações;

- Por falta: usuários que não se encontram no domicílio e/ou não comparecem à Unidade Básica de Saúde e não justificam a ausência após 3 tentativas da eNasf;

- Alta a pedido: usuários que não queiram mais acompanhamento antes do período estabelecido pela eNasf (antes do alcance dos objetivos funcionais estabelecidos) por falta de desejo em ter o acompanhamento, mudança, adesão a outro serviço, entre outros.

\section{DISCUSSÃO}

O uso da CIF como norteadora da construção do instrumento favoreceu uma linguagem comum a todas as profissionais da respectiva eNasf, podendo ser aplicável a maioria das demandas assistenciais acompanhadas no território. Isso se deve ao caráter universal da CIF por considerar a relação entre as atividades e participação do sujeito com as alterações em suas estruturas e funções corporais, sendo também agregado nessa análise o contexto ambiental, físico e social ${ }^{14}$. Dessa forma, percebe-se uma aproximação com os pressupostos da $A B$, pelos quais se busca compreender além do modelo biomédico, com o olhar sobre a multicausalidade de fatores que podem levar às incapacidades ${ }^{15}$.

A elaboração coletiva do instrumento favoreceu a perspectiva interdisciplinar desde a eleição dos códigos da CIF até o tipo de acompanhamento, pois permitiu a identificação e análise das principais demandas dos sujeitos e, a partir disso, refletir sobre a contribuição dos núcleos profissionais no projeto terapêutico, norteando as intervenções a serem adotadas e periodicidade de revisão dos casos acompanhados.

O modelo assistencial ampliado de saúde promove o cuidado numa perspectiva multidimensional e de acordo com as necessidades do usuário, devendo ser desenvolvido através de um trabalho multiprofissional e interdisciplinar, construindo coletivamente uma assistência qualificada e humanizada, gerando troca de experiências e novos conhecimentos e produzindo ações mais efetivas nos serviços de saúde. Nesse contexto, o profissional desempenha papel de interlocutor entre usuários e diferentes mecanismos humanos e tecnológicos que podem contribuir para 0 atendimento das demandas sanitárias e epidemiológicas da população ${ }^{7}$.

Outra importante questão referente à sistematização do uso do protocolo baseado na CIF está em permitir o gerenciamento dos casos, evitando o acúmulo das demandas, tanto para a equipe, quanto para um núcleo profissional específico, auxiliando na gestão compartilhada dos casos e na qualificação da tomada de decisão. Na maioria das vezes os casos eram delimitados para áreas específicas como saúde mental e reabilitação. Com o instrumento foi possível observar que as necessidades dos usuários e famílias são muito mais complexas e as áreas citadas cruzam entre si e principalmente com as questões sociais. Refletir sobre essas múltiplas necessidades foi disparador para as possibilidades de articulação com a rede de saúde e da assistência social, visto que usuários com graves incapacidades necessitam também da atenção especializada e de outros setores.

Enquanto ferramenta clínica, a CIF permite estabelecer objetivos de conduta, padronizando desfechos de saúde e monitorando seu progresso; deslocar o foco de atendimento do diagnóstico e tratamento da doença para a capacidade de realização 
de atividades funcionais e melhoria da saúde, pela intervenção sobre fatores ambientais e pessoais; e auxiliar o planejamento em saúde, facilitando a transição dos usuários entre equipes multidisciplinares de diferentes níveis da RAS $^{16}$.

Como citado anteriormente, o instrumento também favoreceu a definição da proposta terapêutica centrada nos indivíduos e da periodicidade dos atendimentos, tornando-se um elemento organizador do processo de trabalho do Nasf-AB, inclusive no que diz respeito a definição da agenda do profissional, diante da multiplicidade de ações possíveis na perspectiva do AM. O espaço protegido para retorno do atendimento auxilia no monitoramento da evolução das demandas e fortalece o vínculo com o usuário assistido. A equipe citada discute e constrói sua agenda semanal nos espaços de reunião Nasf-AB como forma de distribuir e compartilhar as atividades dos profissionais entre os territórios adscritos pelas eSF.

Checklists incorporados à rotina do serviço podem servir como documentação do estado de saúde dos indivíduos, contribuindo no estabelecimento de metas para o plano terapêutico, de modo que profissional e usuário tenham mais claros os objetivos do tratamento, sendo estes mais específicos e significativos para o usuário e contemplando as demandas do serviço. Ademais, os checklists, além de auxiliarem no acompanhamento longitudinal dos usuários, produzindo dados importantes para análise da evolução dos pacientes numa abordagem biopsicossocial, podem servir para 0 planejamento do serviço com base nas necessidades de assistência ofertada, obtendo indicadores de resolutividade dessa assistência e oferecendo suporte à gestão local, ao promover maior visibilidade ao processo de trabalho de diversas áreas ${ }^{17}$.

Outro aspecto relevante que merece destaque é o acompanhamento tipo 2, através do qual os usuários seguem um plano terapêutico pactuado entre estes e profissionais da eNasf, a fim de possibilitar seu tratamento e cuidado no domicílio. Ao se colocar como protagonista do cuidado, através de propostas terapêuticas que contribuam para tomada de decisão frente seu processo de recuperação, o usuário tem preservadas sua autonomia e independência por não se condicionar à assistência de um profissional de saúde. Isto pode ser potencializado pela construção de um processo terapêutico fruto da associação entre o conhecimento científico e o saber do usuário, o qual se torna mais participativo no seu cuidado ${ }^{18}$.

Merecem destaque também os tipos de acompanhamento 3 e 4 , que permitem a aproximação com os cuidadores, os quais muitas vezes são os próprios familiares. Logo, as orientações precisam ser pensadas tanto na perspectiva dos usuários destacados, quanto no autocuidado do cuidador. $\mathrm{Na}$ maioria dos casos, os cuidadores acabam negligenciando a própria saúde e necessitam de um momento individual para escuta qualificada e serem acolhidos nas rotinas das atividades programáticas das Unidades Básicas de Saúde (UBS).

Ao responsabilizar-se pelo cuidado do outro, o cuidador, muitas vezes, renuncia a suas próprias necessidades, o que pode prejudicar seu autocuidado. Soma-se a isso, o acúmulo de atividades, a falta de suporte de outros familiares e dos serviços de saúde e sentimentos de despreparo e impotência, que geram sobrecarga e tensão emocional. Nesse sentido, a atenção ao cuidador pode ser realizada no âmbito da APS, uma vez que suas equipes desenvolvem um cuidado alicerçado no vínculo entre família e profissionais, apropriando-se do uso de tecnologias em saúde, principalmente das tecnologias leves, que podem, através das relações, fortalecer a escuta sensível, o vínculo, o respeito mútuo, a autonomia e as práticas de acolhimento ${ }^{19}$.

O seguimento sistemático dos usuários em condições estáveis ou que não 
conseguem seguir um plano de cuidado causava limitações na cobertura assistencial, dificultando que novas demandas fossem absorvidas pela equipe. Dessa forma, elaborar os critérios de alta foi necessário para permitir um fluxo contínuo dos usuários assistidos, visto a demanda reprimida do território devido a uma rede de serviços que não atende a necessidade da população. Todavia, as altas são dinâmicas, pois as necessidades das pessoas estão sujeitas a mudanças e é possível a eSF solicitar novamente a retaguarda do Nasf-AB. Então esses casos são reavaliados dentro dos critérios e novas definições poderão ser estabelecidas.

\section{A Atenção Domiciliar 1 (AD1),} modalidade na qual se encontram as equipes da APS, frequentemente envolve condições crônicas de saúde, destinando-se a usuários com problemas de saúde controlados ou compensados que necessitam de cuidados de menor complexidade, dentro da capacidade de atendimento das UBS. Faz-se necessário para essa competência superar o desafio de garantir a continuidade e integralidade do cuidado, especialmente diante da dificuldade das equipes de absorverem tal demanda. A AD1 exige a reorganização dos processos de trabalho, salientando a necessidade de planejamento e gestão do cuidado, através de discussão de casos clínicos, programação de condutas, avaliação das demandas e monitoramento; definição de atribuições entre os membros da equipe; mecanismos de acolhimento das demandas não programadas; classificação da complexidade dos casos por critérios de risco e estabelecimento de prioridades; e definição e aplicação de critérios de inclusão, desligamento e alta da AD1 ${ }^{20}$.

Outro ponto importante a ser frisado é o fato de o instrumento elaborado criar pontos de corte que delimitam quais casos devem ficar sob acompanhamento da eSF e/ou eNasf e quais usuários precisam ser referenciados para serviços da atenção especializada, o que permite uma qualificação dos encaminhamentos, evitando um represamento de demandas em filas de espera. A APS deve ser o local onde 0 diagnóstico da maioria das condições de saúde, a identificação de problemas de funcionalidade, o encaminhamento para serviços especializados e a adesão aos planos de tratamento precisam ocorrer. A promoção dessas atribuições nesse nível de atenção pode facilitar uma perspectiva integrada da atenção ao longo da vida, melhorando a funcionalidade e a qualidade de vida da população ${ }^{21}$.

$O$ trabalho do Nasf-AB através do AM complementa o processo de trabalho e aumenta a capacidade de cuidado das eSF, qualificando os encaminhamentos a outros pontos de atenção (microrregulação) e estabelecendo estratégias e critérios para melhoria do fluxo de atendimento e resolutividade em diferentes pontos da RAS ${ }^{22}$. Associado a isso, sabe-se que o Nasf-AB tem potencial para promover uma reorientação da rede assistencial, tanto pelo aumento do escopo de trabalho quanto pela reorganização dos modos de produzir o cuidado na APS, o que possibilita uma delimitação do espaço de atuação, determinando em que medida alguns tipos de ofertas podem ser disponibilizados pelo Nasf-AB ou devem ser desenvolvidas na atenção especializada ${ }^{3}$.

Vale salientar que o instrumento tem como objetivo orientar a conduta, mas não a engessar perante condições particulares/adversas que venham a acontecer com os usuários e famílias. A devolutiva dos casos permanece acontecendo entre a eNasf e eSF, a fim de garantir a clínica ampliada e a coordenação do cuidado. O processo de organização das demandas dos usuários, ocorre através da discussão dos casos acompanhados e para definição das estratégias de cuidado são levadas em consideração a necessidade destes, das famílias e as condições ofertadas pelo 
território visando dessa forma oferecer um cuidado integral.

O modo como o Nasf-AB pode favorecer a coordenação e integração do cuidado envolve especificidades de gestão, elementos normativos, metodológicos, relacionais e estruturais. É necessário construir diretrizes operacionais claras e recursos para sua estruturação, formalizando processos de cooperação e esclarecendo expectativas e responsabilidades dos atores, bem como uma governança que direcione e apoie inovações relativas à colaboração interprofissional e interorganizacional para elaboração de protocolos de acesso/fluxo de encaminhamentos dentro da RAS ${ }^{3}$.

\section{CONCLUSÃO}

Diante do contexto atual, onde há uma ausência de diretrizes e normativas para nortear o processo de trabalho, as eNasf continuam investindo na leitura de seus territórios, na construção de ações colaborativas e na produção de cuidado integral de qualidade para população que assiste. É necessário que as eNasf criem estratégias assistenciais para organização de demandas, fluxos e comunicação em rede, ainda mais quando se trata de evidenciar o impacto que sua atuação tem nos processos de cuidado na APS.

Nesse sentido, o instrumento objeto deste relato, possibilitou a eNasf a construção de um saber multidimensional sobre clínica ampliada e pactuação de apoio baseado na CIF. Foi possível aprimorar a forma de acompanhamento interdisciplinar, na perspectiva da integralidade, da organização do suporte clínico-assistencial, obedecendo aos pressupostos da coordenação e da gestão compartilhada do cuidado, traçando os tipos de acompanhamentos, critérios de encaminhamentos e de alta. A sistematização do uso da CIF através do protocolo proposto pode contribuir para a gestão do suporte clínico-assistencial do Nasf-AB, contribuindo para a organização da demanda, o acompanhamento longitudinal, o monitoramento da evolução do usuário e a tomada de decisão tanto clínica quanto de gestão do serviço, qualificando os fluxos assistenciais dentro e fora da competência de atuação da APS.

Vale salientar que o produto aqui apresentado é fruto de reflexões a partir das práticas cotidianas de uma eNasf, através das vivências que se originam das necessidades do trabalho. A eNasf autora do instrumento considera que a sistematização dessa experiência é um pontapé inicial para a problematização e construção de definições teóricas orientadoras do seu fazer.

Sugere-se que 0 instrumento criado possa ser objeto de validação futura entre especialistas da área, tornando-se uma alternativa para organização de demandas clínico-assistenciais do Nasf-AB. É fundamental que sejam desenvolvidos outros estudos com maior rigor metodológico, gerando evidências científicas a respeito do impacto do Nasf-AB na coordenação assistencial no território e da sua contribuição enquanto equipe de retaguarda para 0 fortalecimento da APS no país.

\section{REFERÊNCIAS}

1. Brocardo, D., Andrade, C. L. T. de, Fausto, M. C. R. \& Lima, S. M. L. Núcleo de Apoio à Saúde da Família (Nasf): panorama nacional a partir de dados do PMAQ. Saúde em Debate 42, 130-144 (2018).

2. Moreira, D. C., Bispo Júnior, J. P., Nery, A. A. \& Cardoso, J. P. Avaliação do trabalho dos Núcleos Ampliados de Saúde da Família e Atenção Báscia (NASF-AB) por usuários, segundo os atributos da atenção primária. Cad. Saude Publica 36, (2020).

3. Melo, E. A., Miranda, L., Silva, A. M. da \& Limeira, R. M. N. Dez anos dos Núcleos de Apoio à Saúde da Família (Nasf): problematizando alguns desafios. Saúde em Debate 42, 328- 
340 (2018).

4. Gonçalves, R. M. de A., Lancman, S., Sznelwar, L. I., Cordone, N. G. \& Barros, J. de O. Estudo do trabalho em Núcleos de Apoio à Saúde da Introdução. Rev. bras. Saúde ocup. 40, 59-74 (2015).

5. Mazza, D. A. A., Carvalho, B. G., Carvalho, M. N. de \& Mendonça, F. de F. Aspectos macro e micropolíticos na organização do trabalho no NASF: o que a produção científica revela? Physis Rev. Saúde Coletiva 30, 1-27 (2020).

6. ARAUjO NETO, J. D. DE, ALBUQUERQUE, I. M. N., LIRA, G. V. \& BOSI, M. L. M. Aspectos restritivos à integralidade nos Núcleos de Apoio à Saúde da Família: o olhar dos stakeholders. Physis Rev. Saúde Coletiva 28, 1-25 (2018).

7. Santos, M. F. N., Friche, A. A. L. \& Lemos, S. M. A. Atenção à pessoa com deficiência: composição das equipes e distribuição geográfica do componente especializado em reabilitação. CoDAS 33, 1-12 (2021).

8. Araujo, L. B., Novakoski, K. R. M., Bastos, M. S. C., Mélo, T. R. \& Israel, V. L. CARACTERIZAÇÃO DO DESENVOLVIMENTO NEUROPSICOMOTOR DE CRIANÇAS ATÉ TRÊS ANOS: O MODELO DA CIF NO CONTEXTO DO NASF. Cad. Bras. Ter. Ocup. 26, 538-557 (2018).

9. Balco, E. M. Uso da Escala WHODAS 2.0 na atenção Primária à Saúde: perspectivas para a prevenção de incapacidades e promoção da funcionalidade humana pela estratégia de saúde da família. (Universidade de São Paulo - Faculdade de Medicina de Ribeirão Preto, 2018).

10. Souza, T. S. \& Medina, M. G. Nasf: fragmentação ou integração do trabalho em saúde na APS? Saúde em Debate 42, 145-158 (2018).

11. Lanza, L. \& Santos, E. R. dos. NÚCLEOS DE APOIO À SAÚDE DA FAMÍLIA - NASF: ESTRATÉGIA DE APOIO MATRICIAL E SUA INSERÇÃO NOS TERRITÓRIOS EM SAÚDE. in TERRITÓRIOS EM DIÁLOGO: OS CHÃOS COMO PONTO DE PARTIDA PARA AS POLÍTICAS SOCIAIS BRASILEIRAS (orgs. Conserva, M. de S., Alves, J. de M. \& Silva, E. L. P. da) 149-164 (EDITORA DO CCTA, 2016).

12. Rocha, L. P. da et al. Organização do processo de trabalho do Núcleo Ampliado de Saúde da Família a partir de um fluxograma de atuação. in Experiências em Educação Permanente em Saúde no Estado de Pernambuco: formação que se constrói em rede (orgs. Santos, J. S. \& Felipe, D. A.) 344-357 (Secretaria de Saúde, 2019).

13. Holliday, O. J. Para sistematizar experiencias. Innovvando (MMA, 2006).

14. Biz, M. C. P. A CIF E SUA IMPORTÂNCIA NAS POLITICAS PÚBLICAS The ICF and its importance in public policies. Rev. CIF Bras. 3, 40-48 (2015).

15. Piexak, D. R., Cezar-Vaz, M. R. \& Bonow, C. A. International Classification of Functioning, Disability and Health: a Content Analysis / Classificação Internacional de Funcionalidade, Incapacidade e Saúde: uma Análise de Conteúdo. Rev. Pesqui. Cuid. é Fundam. Online 11, 363-369 (2019).

16. Araújo Filho, J. C. de. Desenvolvimento de core set da Classificação Internacional de Funcionalidade, Incapacidade e Saúde para adultos em hemodiálise. (Universidade Federal de Pernambuco, 2020).

17. Biz, M. C. P. \& Chun, R. Y. S. O papel Classificação Internacional de Funcionalidade, Incapacidade e Saúde (CIF) para a tomada de decisão na gestão em serviços de saúde. Saúde em Redes 6, 67-86 (2020).

18. Souza, V. de, Lage, É. G., Matozinhos, F. P. \& Abreu, M. N. S. Fatores associados a não efetivação da alta na assistência domiciliar. Acta Paul. Enferm. 32, 624-631 (2019). 
19. Moura, B. M., Santos, L. F., Rezende, F. A. C., Brito, T. R. P. de \& Nunes, D. P. Cuidando dos cuidadores familiares de idosos dependentes: uma proposta de tecnologia de acolhimento / Caring for family caregivers of dependent elderly people: a proposal for welcoming technology. Brazilian J. Heal. Rev. 3, 12059-12079 (2020).

20. Lopes, G. V. D. O., Vilasbôas, A. L. Q. \& Castellanos, M. E. P. Atenção Domiciliar na Estratégia Saúde da Família: avaliação do grau de implantação em Camaçari (BA). Saúde em Debate 41, 241-254 (2017).

21. Cieza, A. et al. Global estimates of the need for rehabilitation based on the Global Burden of Disease study 2019: a systematic analysis for the Global Burden of Disease Study 2019. Lancet 396, 2006-2017 (2020).

22. Ferrer, M. L. P., Silva, A. S. da, Silva, J. R. K. da \& Padula, R. S. Microrregulação do acesso à rede de atenção em fisioterapia: estratégias para a melhoria do fluxo de atendimento em um serviço de atenção secundária. Fisioter. pesqui 22, 223-230 (2015). 
ANEXOS

FIGURA 1. PROCESSO DE REALIZAÇÃO E SISTEMATIZAÇÃO DA EXPERIÊNCIA

\section{Nome do}

projeto/área/progra

ma

\begin{tabular}{l|l} 
Semana 01 & 17 a 21 de dezembro de 2018
\end{tabular}

\section{Protocolo de elegibilidade para avaliação e monitoramento de casos}

\begin{tabular}{|c|c|c|c|c|c|}
\hline $\begin{array}{c}\text { Atividad } \\
\text { e }\end{array}$ & Data & Objetivos & $\begin{array}{c}\text { Responsá } \\
\text { vel }\end{array}$ & $\begin{array}{c}\text { Com } \\
\text { quem }\end{array}$ & Resultados \\
\hline \multirow{2}{*}{$\begin{array}{l}\text { Reunião } \\
\text { de eNasf }\end{array}$} & $\begin{array}{c}18 / 12 / 20 \\
18\end{array}$ & \multirow{2}{*}{$\begin{array}{l}\text { Discutir e analisar a } \\
\text { problemática da } \\
\text { demanda pelo } \\
\text { suporte clínico- } \\
\text { assistencial }\end{array}$} & \multirow{2}{*}{ eNasf } & \multirow{2}{*}{$\begin{array}{c}\text { Profissiona } \\
\text { is da } \\
\text { equipe }\end{array}$} & \multirow{2}{*}{$\begin{array}{l}\text { Falta de definição do modo de acompanhamento do } \\
\text { usuário no território; variedade na forma de condução } \\
\text { dos casos entre os profissionais; grande número de } \\
\text { usuários em acompanhamento, descontinuidade no } \\
\text { cuidado. }\end{array}$} \\
\hline & $\begin{array}{c}20 / 12 / 20 \\
18\end{array}$ & & & & \\
\hline \multicolumn{2}{|c|}{ Semana 02} & \multicolumn{4}{|c|}{26 a 28 de dezembro de 2018} \\
\hline $\begin{array}{c}\text { Atividad } \\
\mathrm{e}\end{array}$ & Data & Objetivos & $\begin{array}{c}\text { Responsá } \\
\text { vel }\end{array}$ & $\begin{array}{l}\text { Com } \\
\text { quem }\end{array}$ & Resultados \\
\hline $\begin{array}{l}\text { Reunião } \\
\text { de eNasf }\end{array}$ & $\begin{array}{c}26 / 12 / 20 \\
18\end{array}$ & \multirow{2}{*}{$\begin{array}{c}\text { Realizar análise } \\
\text { sistêmica das ações } \\
\text { clínico-assistenciais } \\
\text { e propor } \\
\text { instrumento de } \\
\text { avaliação e } \\
\text { monitoramento }\end{array}$} & eNasf & $\begin{array}{c}\text { Profissiona } \\
\text { is da } \\
\text { equipe }\end{array}$ & $\begin{array}{c}\text { Necessidade de elaboração de instrumento de } \\
\text { avaliação e monitoramento de fácil aplicação, nas } \\
\text { prerrogativas da CA, contemplando as diferentes } \\
\text { especialidades da equipe, a fim de criar critérios de } \\
\text { acompanhamento, continuidade da assistência alta e } \\
\text { encaminhamento dos casos. }\end{array}$ \\
\hline $\begin{array}{l}\text { Reunião } \\
\text { de eNasf }\end{array}$ & $\begin{array}{c}26 / 12 / 20 \\
18\end{array}$ & & eNasf & $\begin{array}{c}\text { Profissiona } \\
\text { is da } \\
\text { equipe }\end{array}$ & $\begin{array}{l}\text { Necessidade de estudo direcionado para a } \\
\text { problemática, buscando referências, instrumentos e } \\
\text { normativas que pudessem embasar a elaboração do } \\
\text { instrumento de avaliação e monitoramento. }\end{array}$ \\
\hline \multicolumn{2}{|c|}{ Semana 03} & \multicolumn{4}{|c|}{02 a 04 de janeiro de 2019} \\
\hline
\end{tabular}




\begin{tabular}{|c|c|c|c|c|c|}
\hline $\begin{array}{c}\text { Atividad } \\
\mathrm{e}\end{array}$ & Data & Objetivos & $\begin{array}{c}\text { Responsá } \\
\text { vel }\end{array}$ & $\begin{array}{l}\text { Com } \\
\text { quem }\end{array}$ & Resultados \\
\hline $\begin{array}{c}\text { Estudos } \\
\text { individuai } \\
\mathrm{s}\end{array}$ & $\begin{array}{c}02,03 \text { e } \\
04 / 01 / 20 \\
19\end{array}$ & $\begin{array}{l}\text { Buscar referências, } \\
\text { instrumentos e } \\
\text { normativas para } \\
\text { propor instrumento } \\
\text { de avaliação e } \\
\text { monitoramento }\end{array}$ & eNasf & $\begin{array}{c}\text { Profissiona } \\
\text { is da } \\
\text { equipe }\end{array}$ & $\begin{array}{l}\text { Mapeamento de possíveis ferramentas e instrumentos } \\
\text { de avaliação e monitoramento: avaliação de qualidade } \\
\text { de vida (SF-36); inventário de depressão de Beck; mini- } \\
\text { mental; medida de independência funcional (MIF); } \\
\text { escala de Barthel; Índice de Katz; CIF. }\end{array}$ \\
\hline \multicolumn{2}{|c|}{ Semana 04} & \multicolumn{4}{|c|}{07 a 11 de janeiro de 2019} \\
\hline $\begin{array}{c}\text { Atividad } \\
\mathbf{e}\end{array}$ & Data & Objetivos & $\begin{array}{c}\text { Responsá } \\
\text { vel }\end{array}$ & $\begin{array}{l}\text { Com } \\
\text { quem }\end{array}$ & Resultados \\
\hline $\begin{array}{l}\text { Reunião } \\
\text { de eNasf }\end{array}$ & $\begin{array}{c}07 / 01 / 20 \\
19\end{array}$ & $\begin{array}{l}\text { Elencar e/ou } \\
\text { elaborar } \\
\text { instrumento de } \\
\text { avaliação e } \\
\text { monitoramento }\end{array}$ & eNasf & $\begin{array}{c}\text { Profissiona } \\
\text { is da } \\
\text { equipe }\end{array}$ & $\begin{array}{c}\text { Eleição da CIF como base para elaboração do } \\
\text { instrumento de avaliação e monitoramento; necessidade } \\
\text { de matriciamento para nivelamento dos conhecimentos } \\
\text { sobre a CIF entre a eNasf. }\end{array}$ \\
\hline $\begin{array}{l}\text { Reunião } \\
\text { de eNasf }\end{array}$ & $\begin{array}{c}09 / 01 / 20 \\
19\end{array}$ & $\begin{array}{c}\text { Apresentar a CIF e } \\
\text { nivelar } \\
\text { conhecimentos } \\
\text { sobre a ferramenta } \\
\text { entre profissionais } \\
\text { da eNasf }\end{array}$ & $\begin{array}{l}\text { Terapeuta } \\
\text { Ocupacion } \\
\text { al da } \\
\text { eNasf }\end{array}$ & $\begin{array}{c}\text { Profissiona } \\
\text { is da } \\
\text { equipe }\end{array}$ & $\begin{array}{l}\text { Nivelamento dos conhecimentos sobre a CIF entre a } \\
\text { eNasf e indicativo de estudo individual para eleição de } \\
\text { códigos que pudessem compor o instrumento de } \\
\text { avaliação e monitoramento. }\end{array}$ \\
\hline $\begin{array}{l}\text { Estudo } \\
\text { individual } \\
\text { de cada } \\
\text { profission } \\
\quad \text { al }\end{array}$ & $\begin{array}{c}11 / 01 / 20 \\
19\end{array}$ & $\begin{array}{l}\text { Eleger códigos da } \\
\text { CIF representantes } \\
\text { de cada categoria } \\
\text { profissional no } \\
\text { instrumento }\end{array}$ & eNasf & $\begin{array}{c}\text { Profissiona } \\
\text { is da } \\
\text { equipe }\end{array}$ & $\begin{array}{l}\text { Códigos eleitos: b114, b134, b144, b152, b167, b230, } \\
\text { b280, b510, b530, b710, d240, d410, d450, d470, d510, } \\
\text { d540, d550, d570, d710, d920, d640, d760, d845, e325, } \\
\text { e5750, e5800, e5850 }\end{array}$ \\
\hline
\end{tabular}




\begin{tabular}{|c|c|c|c|c|c|}
\hline $\begin{array}{c}\text { Atividad } \\
\text { e }\end{array}$ & Data & Objetivos & $\begin{array}{l}\text { Responsá } \\
\text { vel }\end{array}$ & $\begin{array}{c}\text { Com } \\
\text { quem }\end{array}$ & Resultados \\
\hline \multirow{2}{*}{$\begin{array}{l}\text { Reunião } \\
\text { de eNasf }\end{array}$} & $\begin{array}{c}15 / 01 / 20 \\
19\end{array}$ & $\begin{array}{l}\text { Construir } \\
\text { instrumento de }\end{array}$ & eNasf & $\begin{array}{c}\text { Profissiona } \\
\text { is da }\end{array}$ & \multirow{2}{*}{$\begin{array}{l}\text { Produção da versão preliminar do instrumento de } \\
\text { avaliação e monitoramento baseado na CIF }\end{array}$} \\
\hline & $\begin{array}{c}17 / 01 / 20 \\
19\end{array}$ & $\begin{array}{c}\text { avaliação e } \\
\text { monitoramento }\end{array}$ & & equipe & \\
\hline \multicolumn{2}{|c|}{ Semana 07} & \multicolumn{4}{|c|}{21 a 25 de janeiro de 2019} \\
\hline $\begin{array}{c}\text { Atividad } \\
\text { e }\end{array}$ & Data & Objetivos & $\begin{array}{c}\text { Responsá } \\
\text { vel }\end{array}$ & $\begin{array}{l}\text { Com } \\
\text { quem }\end{array}$ & Resultados \\
\hline $\begin{array}{l}\text { Reunião } \\
\text { de eNasf }\end{array}$ & $\begin{array}{l}22 / 01 / 20 \\
19\end{array}$ & $\begin{array}{l}\text { Estabelecer tipos de } \\
\text { acompanhamento, } \\
\text { critérios de } \\
\text { encaminhamento e } \\
\text { alta }\end{array}$ & eNasf & $\begin{array}{l}\text { Profissiona } \\
\text { is da } \\
\text { equipe }\end{array}$ & $\begin{array}{l}\text { Estabelecimento de tipos de acompanhamento; } \\
\text { construção dos critérios de encaminhamento e alta. }\end{array}$ \\
\hline \multicolumn{2}{|c|}{ Semana 08} & \multicolumn{4}{|c|}{28 de janeiro a 01 de fevereiro de 2019} \\
\hline $\begin{array}{l}\text { Atividad } \\
\text { e }\end{array}$ & Data & Objetivos & $\begin{array}{l}\text { Responsá } \\
\text { vel }\end{array}$ & $\begin{array}{l}\text { Com } \\
\text { quem }\end{array}$ & Resultados \\
\hline $\begin{array}{l}\text { Reunião } \\
\text { de eNasf }\end{array}$ & $\begin{array}{l}29 / 01 / 20 \\
19\end{array}$ & $\begin{array}{l}\text { Elaborar fluxograma } \\
\text { de decisão para } \\
\text { seguimento dos } \\
\text { casos }\end{array}$ & eNasf & $\begin{array}{l}\text { Profissiona } \\
\text { is da } \\
\text { equipe }\end{array}$ & $\begin{array}{l}\text { Primeira versão de fluxograma de decisão com scores e } \\
\text { pontos de corte para orientação quanto ao tipo de } \\
\text { acompanhamento dos casos e a necessidade } \\
\text { encaminhamento para outros pontos da RAS. }\end{array}$ \\
\hline \multicolumn{2}{|c|}{ Semanas 09,10 e 11} & \multicolumn{4}{|c|}{28 de janeiro a 01 de fevereiro de 2019 | 04 a 08 de fevereiro de 2019 | 11 a 15 de fevereiro de 2019} \\
\hline $\begin{array}{c}\text { Atividad } \\
\text { e }\end{array}$ & Data & Objetivos & $\begin{array}{l}\text { Responsá } \\
\text { vel }\end{array}$ & $\begin{array}{l}\text { Com } \\
\text { quem }\end{array}$ & Resultados \\
\hline Pilotos & $\begin{array}{l}\text { Ao longo } \\
\text { das } \\
\text { semanas }\end{array}$ & $\begin{array}{c}\text { Testar instrumento } \\
\text { de avaliação e }\end{array}$ & eNasf & $\begin{array}{l}\text { Usuários } \\
\text { com } \\
\text { demanda }\end{array}$ & $\begin{array}{c}\text { Necessidade de adaptações nos escores do fluxograma } \\
\text { de decisão e elaboração de outras três versões para } \\
\text { correções }\end{array}$ \\
\hline
\end{tabular}




\begin{tabular}{|c|c|c|c|c|c|}
\hline Semana 12 & $\begin{array}{c}\text { monitoramento e } \\
\text { fluxograma proposto }\end{array}$ & $\begin{array}{c}\text { para o } \\
\text { Nasf }\end{array}$ & \multicolumn{2}{|c|}{ Resultados } \\
\hline $\begin{array}{c}\text { Atividad } \\
\text { e }\end{array}$ & Data & Objetivos & $\begin{array}{c}\text { Responsá } \\
\text { vel }\end{array}$ & $\begin{array}{c}\text { Com } \\
\text { quem }\end{array}$ & fevereiro de 2019 \\
\hline $\begin{array}{c}\text { Reunião } \\
\text { de eNasf }\end{array}$ & $\begin{array}{c}19 / 02 / 20 \\
19\end{array}$ & $\begin{array}{c}\text { Adaptar versão final } \\
\text { do fluxograma de } \\
\text { decisão }\end{array}$ & eNasf & $\begin{array}{c}\text { Profissiona } \\
\text { is da } \\
\text { equipe }\end{array}$ & $\begin{array}{c}\text { Versão final do instrumento e do fluxograma de decisão: } \\
\text { Protocolo de elegibilidade para avaliação e } \\
\text { monitoramento de casos }\end{array}$ \\
\hline
\end{tabular}

Fonte: elaborado pelas autoras, adaptado de Holliday, 2019 
FIGURA 2. PROTOCOLO DE ELEGIBILIDADE PARA AVALIAÇÃO E MONITORAMENTO DE CASOS

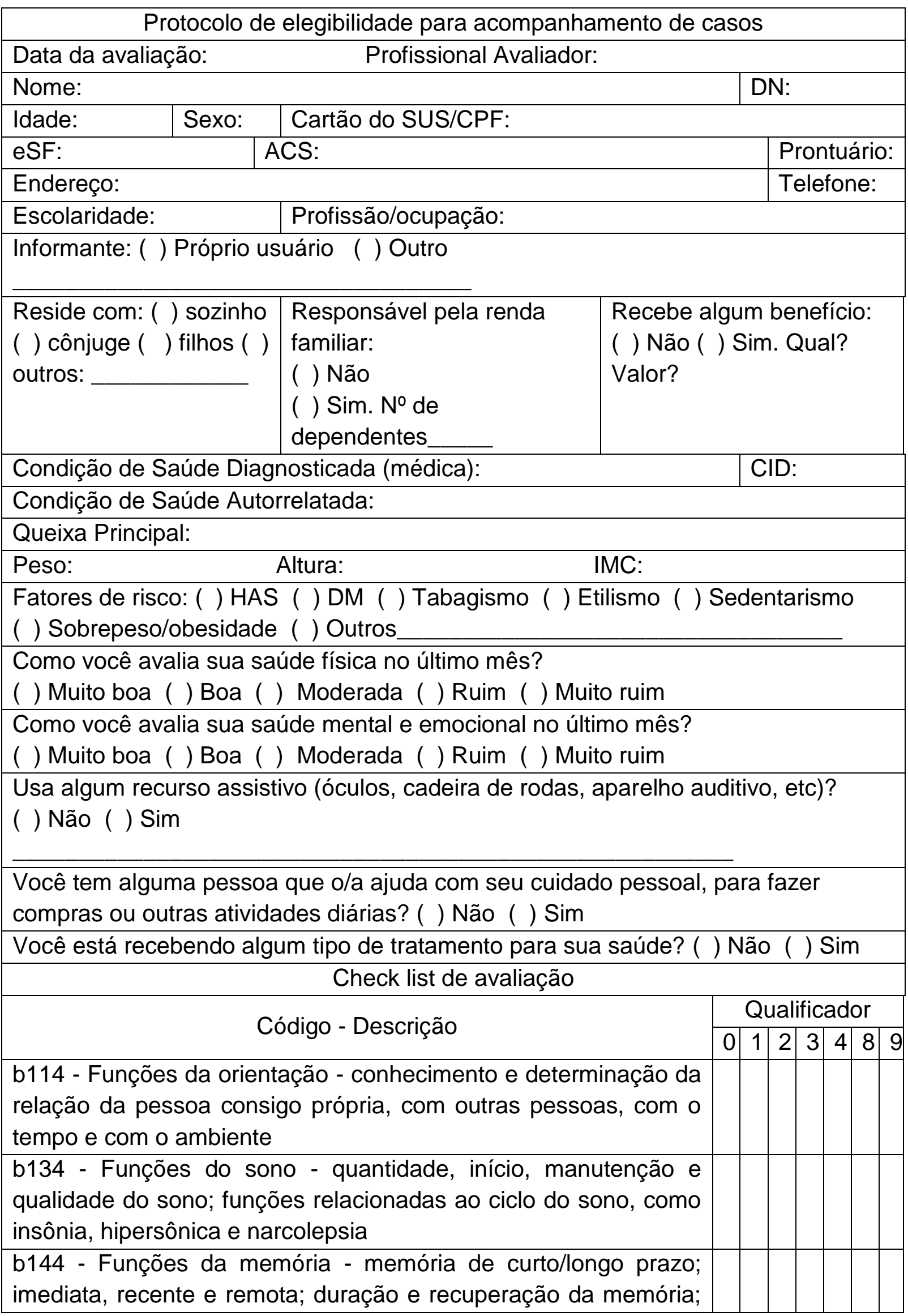




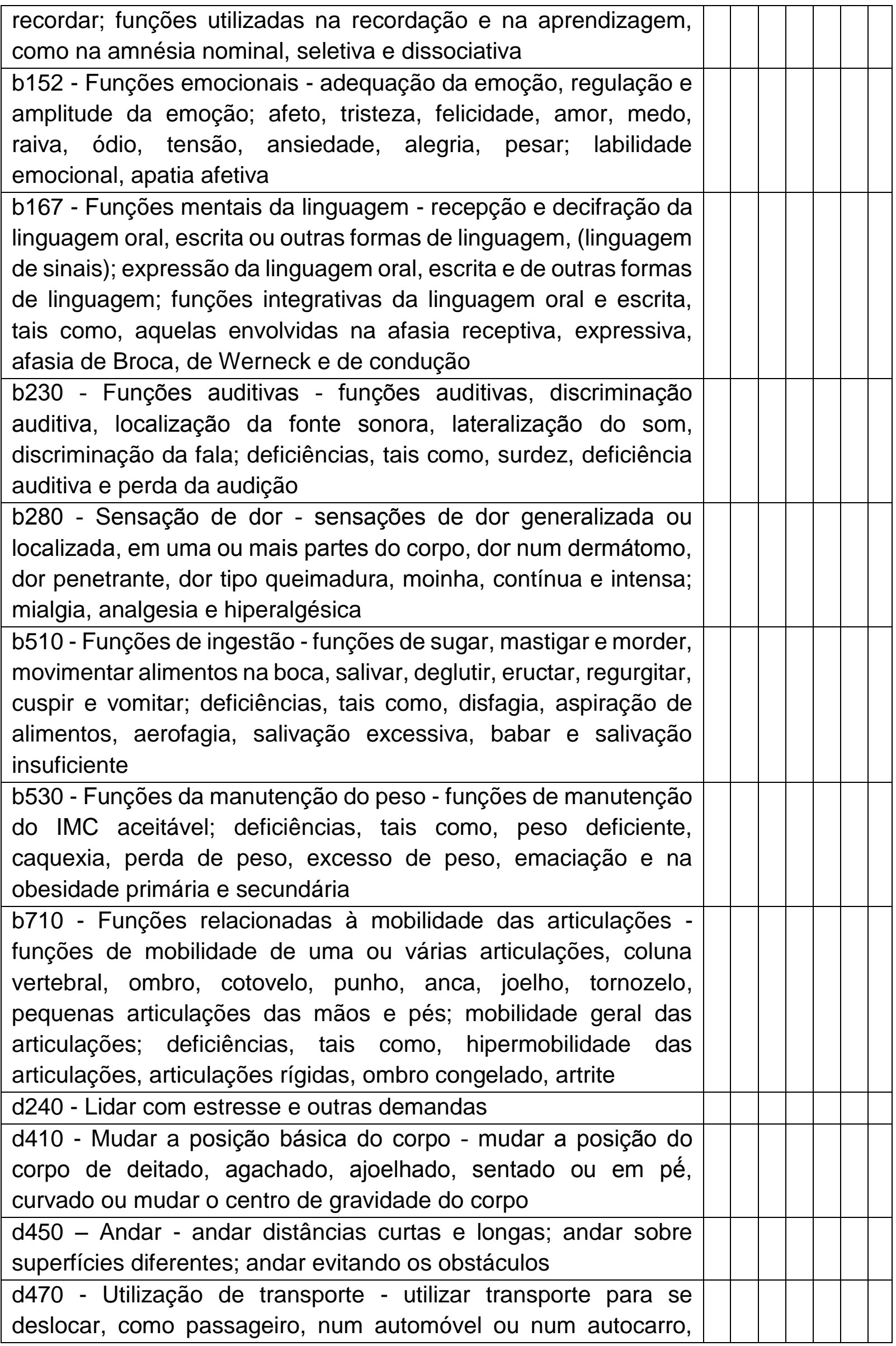




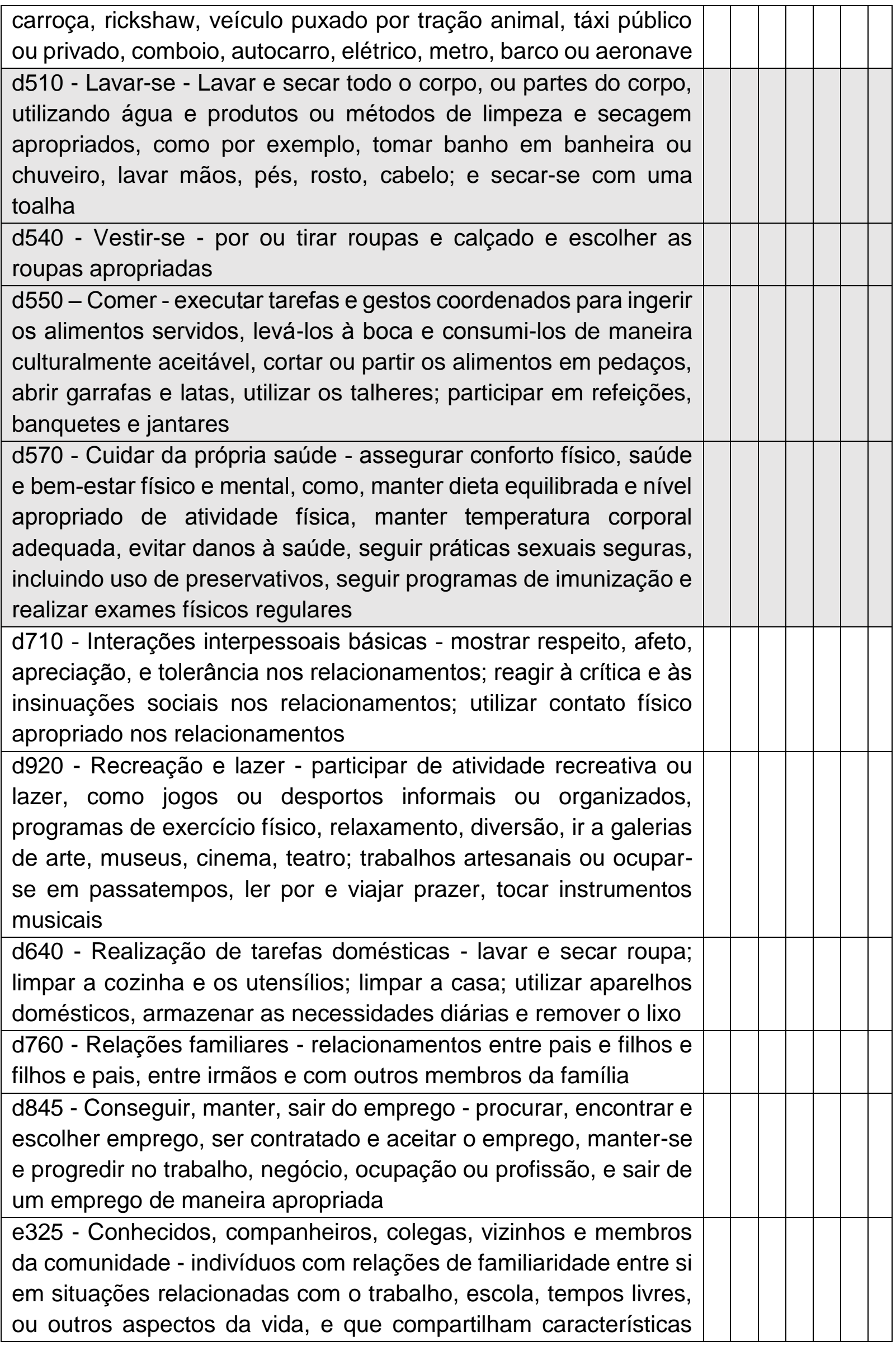




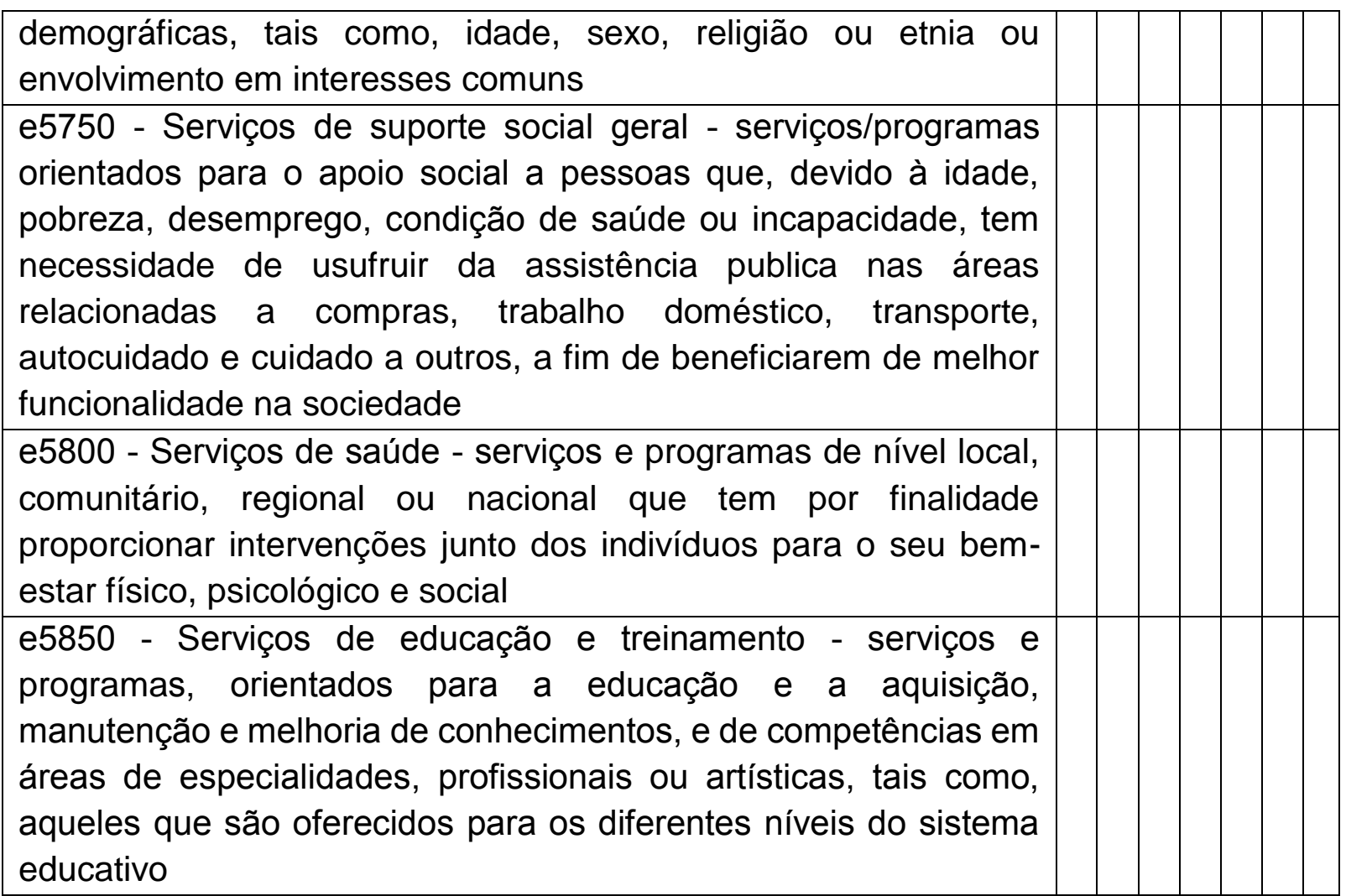

Fonte: elaborado pelas autoras, 2019 
FIGURA 3. FLUXOGRAMA DE DECISÃO PARA ELEIÇÃO DO TIPO DE ACOMPANHAMENTO DO USUÁRIO

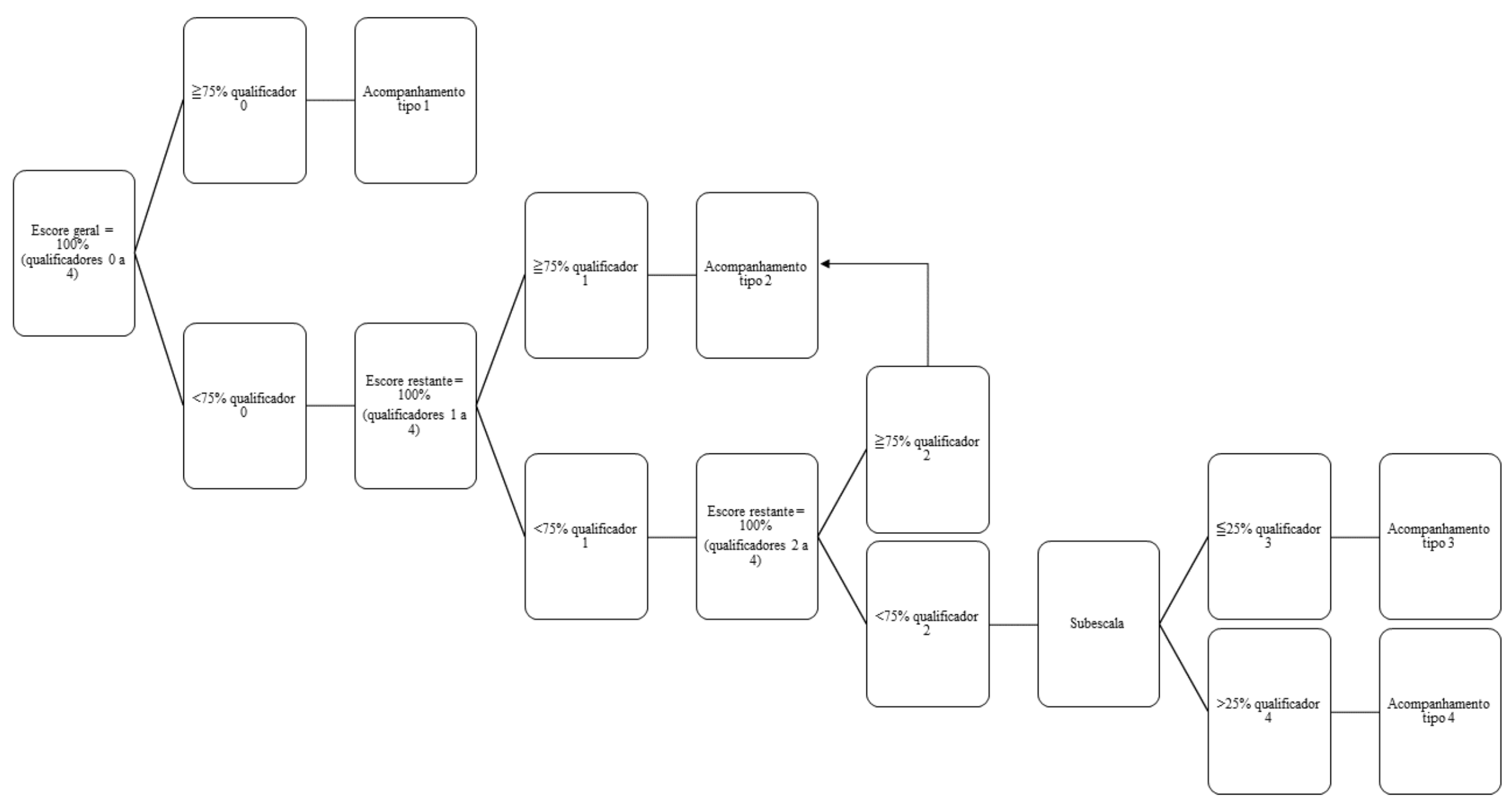

Fonte: elaborado pelas autoras, 2019 